\title{
Carbon ceramics from plants: Graphitization of biomorphic matrixes
}

\author{
D.A. Iarmolenko, A.E. Belyaev, V.S. Kiselov \\ V. Lashkaryov Institute of Semiconductor Physics, NAS of Ukraine; \\ 41, prospect Nauky, 03028 Kyiv, Ukraine \\ Corresponding author’s e-mail: iarmolenko.dmytro@isp.kiev.ua
}

\begin{abstract}
Properties of matrixes obtained from plants at various pyrolysis temperatures have been discussed. The article is devoted to graphitization of carbon matrixes obtained from plants. All stages of production, starting from preparation of the precursors up to high-temperature pyrolysis, have been considered together with some specific properties of matrixes at each stage. Previously unexplored new structural surface changes have been found. The use of potassium-doped wooden precursors showed the graphitization property of the matrix surfaces and allowed to form the matrix capillary wall coating with layers of crystalline graphite as well as graphene flakes and films with a low number of defects. New possibilities to apply these matrixes as examples of ultracapacitor, power unit filter electromagnetic interference and audio power amplifier antiresonance element have been discussed as well.
\end{abstract}

Keywords: carbon ceramics, biomorphic graphite, graphitization, potassium-doped glassy carbon, biomorphic grapheme.

Manuscript received 18.11.15; revised version received 27.01.16; accepted for publication 16.03.16; published online 08.04.16.

\section{Introduction}

Pyrolysis of plants and seeds [1] depending on the process temperature [2] provides with a wide range of materials having various properties. Being obtained from various species of plants and seeds, they are a promising basis to replace carbon fillers, threads and tissues in production of composite heat-resistant and heat-stressed materials, chemical and thermoelectric current source electrodes, catalytic carriers, catalytic supporters, filters and sorbents. In a number of cases, application of plant matrixes reduces costs for development and production due to replacement of 3D-6D reinforcement complex tying system with mechanical processing of porous matrixes. This is an essential factor of selection or design of material properties. Porous electrodes made of graphitized matrixes feature high penetrability and material purity, adjustable anisotropic porosity, and can serve as catalytic supporters and sorbents possessing the effectiveness comparable with that of nanopowder ones, and combine the further filtration function.

Some of the plant matrix properties are inherited from carbon in its graphite allotropic modification, namely: electrical conductivity, absence of forbidden band, resistance to high temperatures and high specific strength. The other group of the properties is inherited from the cell structure depending on the respective vital functions.

Porosity requires separate consideration. Plant matrixes have highly developed capillary and porous structure. The physical and mechanical properties depend on the type of processing and preparing the 
precursors, the plant species as well as the part that the tissues consist of; the latter are classified as storage, conductive, supporting and integumentary tissues [3]. Matrixes of this type feature anisotropy. Thus, electrical conductivity, thermal conductivity, penetrability and wettability, mechanical compression, torsional, tensile strength and impact elasticity sharply vary along and across the capillaries.

\section{State of the art}

In the study [4], the pyrolysis method at $1800{ }^{\circ} \mathrm{C}$ was described. These samples contained no excessive silicon and could be filled when necessary. It was reported about the possibility to use anisotropic cellular ceramics in technical applications such as heat-insulating structures, filters and catalytic supporters at high temperatures, thermally and mechanically stressed light structures as well as medical implants.

In the work [5], mechanical properties and microstructures of silicon carbide ceramics of wooden carbon precursors were studied. To obtain matrixes, temperatures not exceeding $1000{ }^{\circ} \mathrm{C}$ were used [4].

In the study [6], thermoelectric and electric properties of silicon carbide on silicon substrate and biomorphic composite were studied. The electric resistance within 100-950 K range declined along with temperature increase. Thermal electromotive force rose virtually linearly within the range 100 to $750 \mathrm{~K}$. Pyrolysis took place in argon at the temperature of $1000{ }^{\circ} \mathrm{C}$. After impregnation, the samples contained excess silicon up to $30 \%$ with residual porosity up to $15 \%$.

Matrixes obtained at temperatures not exceeding $1000{ }^{\circ} \mathrm{C}$ contain rather large amount of impurities in the form of metals and their compounds, namely: calcium and potassium, compounds of nitrogen and glassy carbon; the carbon order degree is low. It complicates impregnation of matrixes with silicon and prevents from uniform filling; in order to solve this problem, frequently forced impregnation is used, which results in silicon excess.

In the work [7], red cedar was pyrolyzed at $750{ }^{\circ} \mathrm{C}$ in nitric atmosphere in order to produce ultracapacitor electrodes. The obtained specific electric capacitance was $14 \mathrm{~F} / \mathrm{g}$, and after electrode activation with nitric acid, the capacitance was $115 \mathrm{~F} / \mathrm{g}$; the used electrolyte was sulphuric acid aqueous solution. It was also noted that electrode formation took place within one stage, which seems easier than in traditional technology.

In most cases, the source materials for obtaining matrixes are solid wood types, the share of which in gross plant mass is low. A good alternative may be fruits, seeds, annual plants, leafage, needles and algae; the best hardness and porosity properties are considered to feature coconut shell.

The energy obtained by burning of pyrolyzed gases is not sufficient to achieve the process temperatures over
$1000{ }^{\circ} \mathrm{C}$, but the advantages of this method include low and zero energy losses as well as no need to use protective inert gases.

'Low-temperature' matrixes feature high electric resistance and sensitivity to heating and cooling speeds, low carbon order degree, long formation time and high impurity content.

Obtaining the high-quality matrixes requires stoves with uniform thermal fields, since non-uniform heating results in formation of inner tensions, fractures and fissures as well as different shrinkage time. Shrinkage in different directions is always non-uniform, is difficult enough for calculation and occurs only once within the entire pyrolysis temperature range. A remarkable property is that the matrix is almost subjected to no changes until temperature exceeds a value once achieved.

The purpose of this paper is development of technology for obtaining a carbonized material with high electric conduction and higher surface area, suitability for being used as power electrodes as well as a study of the influence of pyrolysis temperature on electric resistance of biomorphic carbon matrixes and assessment of their applicability as electrodes for double electron layer capacitors.

\section{Materials and experimental details}

Depending on the source material and final desired properties of matrixes to be obtained, stove structures and technological operations may significantly differ, but physical and chemical processes remain the same.

Provisionally, the entire process may be separated by the following stages:

1) Source material must be prepared. Wood may require moisture adjustment, preliminary structure compaction, cutting into billets of required size and shape; grassy plants, leafs and needles require refinement and certain orientation arrangement, probably, tying, gluing, pressing, preliminary forming and drying.

2) The most intensive release of pyrolysis products takes place within the range $200 \ldots 400{ }^{\circ} \mathrm{C}$. At these temperatures, electric conduction is not observed. The pyrolysis products are released both at the gaseous and liquid phases. The process speed is not high, since the source material has a low heat conductivity. Temperature must be increased slowly. The temperature gradient inside the material must not be large in order that the shrinkage process may develop uniformly in the entire volume. In this mode, pyrolysis with thermally activated plastic compaction of wood or another source material does not meet the desired requirements. Thus, in our laboratory, the hornbeam matrix was compacted 4.5 times along one axis, i.e. along fibers, and the matrix density was $2.05 \mathrm{~g} / \mathrm{cm}^{3}$. The 'tablet' retained its structure. 
Obviously, there is no wood with the initial density of $3 \ldots 3.5 \mathrm{~g} / \mathrm{cm}^{3}$ to obtain a matrix with the density close to 2 . The cell wall material density is almost the same for all wood species, being $1.54 \mathrm{~g} / \mathrm{cm}^{3}$. The purpose of this experiment was shortening the transport duct for electrolyte in the ultracapacitor electrode.

3) At $400 \ldots 700^{\circ} \mathrm{C}$, pyrolysis product emission intensity lowers; electric conduction of the carbon structure appears; heating and cooling at this stage must be slow, as the amount of glassy hydrocarbon inclusions is large enough.

4) At $700 \ldots 1000^{\circ} \mathrm{C}$, electricity-conductive properties improve; at this stage, coal activation by steam, adjustment of wall thickness and surface area may be used with moderate heating and cooling rates.

5) At $1000 \ldots 2000{ }^{\circ} \mathrm{C}$, melting-out of salts and oxides and minor shrinkage take place; the carbon structure virtually does not change; the heating rate may range from moderate to high.

6) At $2000 \ldots 4000{ }^{\circ} \mathrm{C}$, sublimation of salts and melting of acetylides takes place; at the end of the range, boiling of acetylides, formation of ordered carbon in the form of graphite and graphene surfaces occurs; shrinkage may achieve 30\% and more; the heating and cooling rates may be very high. The obtained samples feature low impurity content.

For pyrolysis with thermally activated plastic compaction, a vertical tubular stainless steel stove with a fired heater and a thermocouple thermometer was used. The upper part of the stove was equipped with a perforated pressure dome with a silicon oxide filling shutter situated above. The inlet spout returned gases to the fired heater area through the distributor. In the lower part, there was a movable pressure dome, on which the pressure pusher acted during the pyrolysis process. The lower pressure dome was equipped with an outlet spout to discharge liquid pyrolysis products through the water valve.

To obtain temperatures up to $1000{ }^{\circ} \mathrm{C}, 800-\mathrm{mm}$ long silica tubular stove with a resistance-type heater, a heating current controller and a thermostat were used. The stove was equipped with inlet and outlet spouts to create nitric atmosphere and water steam supply, and one more outlet spout for discharge of pyrolysis products through the water valve. The pictures show a typical hornbeam matrix (Fig. 1A) in the scale 100×.

To obtain higher temperatures, a direct-heating stove was used. The stove current was within the range $0.4 \ldots 4 \mathrm{kA}$; the process lasted until the current limit switch triggering. The stove was a stainless steel cylinder. The side wall has an inspection window for pyrometric temperature measurements. Inside the cylinder, immobile and mobile current leads were placed coaxially. The stove was equipped with an inlet spout in order to create nitric atmosphere and an outlet one for discharge of the pyrolysis products through the water valve. The pictures show a typical hornbeam matrix along (Fig. 1B) and across (Fig. 1C) fibers, 100× scale.
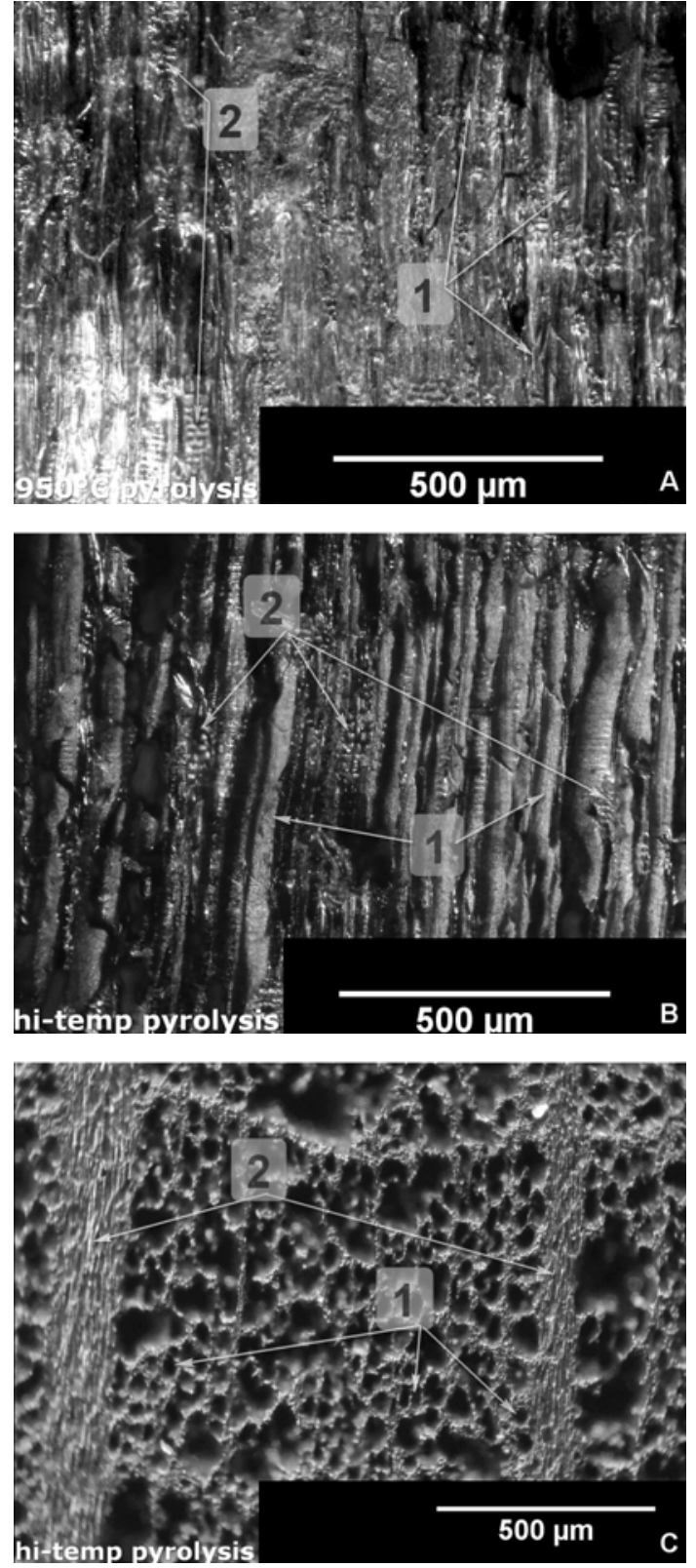

Fig. 1. Hornbeam matrix. A) Matrix cleavage along the fibers, glossy amorphous glassy carbon capillary surface. B) Matrix cleavage along the fibers, crystalline graphite capillary surface. C) Transverse section of the matrix in scattered light; the filled areas are transverse wooden fibers: 1 - longitudinal fibers with the typical diameter about $50 \mu \mathrm{m} ; 2$ - longitudinal fibers with the typical diameter about $10 \mu \mathrm{m}$.

Electric resistance was measured by voltage drop on the sample, within the circuit with a direct current source. To include the samples into the circuit, pincers with smooth graphite sponges and fixed pressing force were used. Before measurements, the ends of the samples were grinded on a mandrel for plane-parallelism. After grinding, the ends were cleaned by an electric-discharge machine. The measurement results varied significantly from sample to sample: 2 to 3 times from one pyrolysis loading and in different loadings. It is

\section{(C) 2016, V. Lashkaryov Institute of Semiconductor Physics, National Academy of Sciences of Ukraine}


conditioned by the presence of inner ruptures. The resistance of the best samples in various loadings was $30 \ldots 35$ milliohms. It is a very good result in comparison with foreign analogues, but design resistance of a graphite rod of the same length and weight shows 20 times lower resistance value. It was a starting point for pyrolysis at $2000{ }^{\circ} \mathrm{C}$ and higher temperatures. But the sources $[8,9]$ contain information that not every carbonbase material is able to graphitize even at very high temperatures through the presence of nitrogen and oxygen bridges between disordered carbon clusters, which prevents from formation of extensive graphite structures. However, it was not proved for biomorphic carbon matrixes. According to the results of Raman spectroscopy, not only graphite but graphene forms.

The sample weight and size were also measured before and after pyrolysis. For $950{ }^{\circ} \mathrm{C}$ pyrolysis, average weight loss was $80 \%$ with average shrinkage of $65 \%$ in volume.

For the ultracapacitor, a model of symmetric double electron layer on acid aqueous electrolyte was chosen. A low-resistant carbon matrix was selected from the samples that had undergone $950{ }^{\circ} \mathrm{C}$ pyrolysis. The current collectors were made of lead. The electrolyte
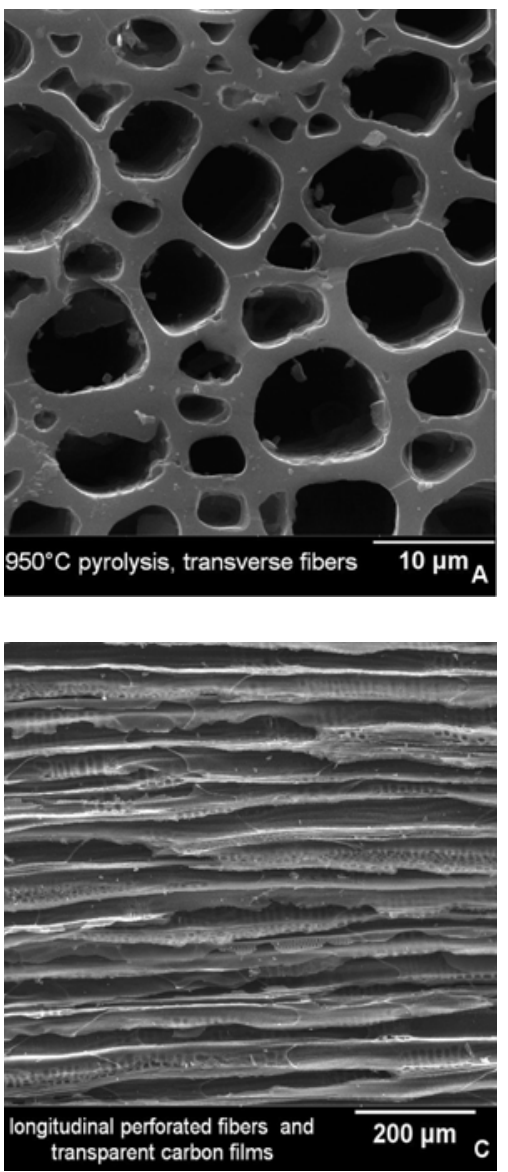

was the sulphuric acid aqueous solution with density of 1.27. The current leads were made using the mould casting method. Any separator was not used; instead of it, 2 glass spacers were installed, which ensured $0.5-\mathrm{mm}$ space between the matrixes.

Using a scanning electron microscope, the samples made of one solid wood were examined. A sample cleavage was taken for examination. This operation opens the access to examination of the capillary skeleton and surface. The skeleton element composition includes oxygen up to $3.4 \%$ and carbon about $96.6 \%$. The capillary surface is $100 \%$ carbon. On the surface of some capillaries, numerous graphene flakes (Fig. 2B) with a typical size of about $2 \mu \mathrm{m}$ were found. In addition, at the places of ruptures caused by the cleavage operation, graphene films were found. The film edges were ragged; some films were transparent for electrons (Fig. 2C, D); there were no visible defects on the surface; other objects could be seen well through them; the film composition was $100 \%$ carbon. The element composition of the samples greatly varied depending on the pyrolysis temperature as well as the surface composition of the capillaries and the skeleton within one sample. As the temperature rose, the samples were carbonized and purified.
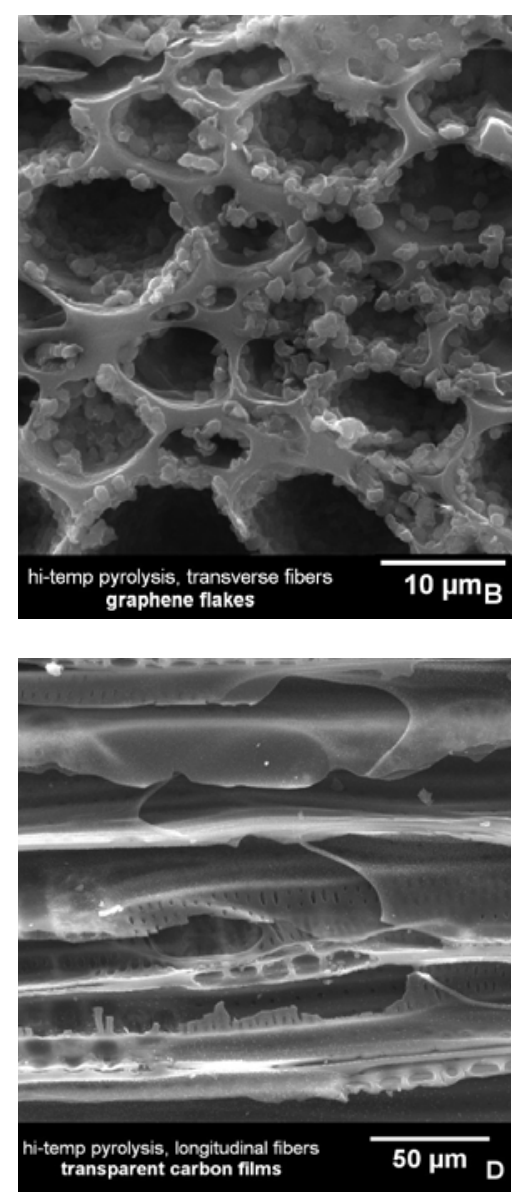

Fig. 2. Scanning electron microscope images. A) Transverse cleavage of transverse fibers of the glassy carbon matrix with thick walls. B) Formation of graphene flakes exhausts the capillary walls. C) Cleavage along the longitudinal fibers; capillary walls are covered with graphene crystalline films; D) Multilayer films with cleaved edges, the films are transparent, perforated capillary surfaces can be seen through them, longitudinal fiber perforation is junctions with transverse capillaries.

\section{(C) 2016, V. Lashkaryov Institute of Semiconductor Physics, National Academy of Sciences of Ukraine}


Raman scattering spectra were measured in backscattering geometry at room temperature. For excitation, 514.5-nm wavelength laser was used; radiation was focused within the $1-\mu \mathrm{m}$ spot; the incident radiation power was close to $1 \mathrm{~mW}$.

The spectra are typical for crystalline graphite (Fig. 3A), graphene flakes (Fig. 3B) and graphene with a low number of defects (Fig. 3C).

The optical microscopy was made with $40 \times$ and $100 \times$ magnifications.

With $100 \times$ magnification, orbicular objects can be seen (Fig. 4A). In contrast to matrixes prepared at temperatures close to $1000{ }^{\circ} \mathrm{C}$, in the transverse section
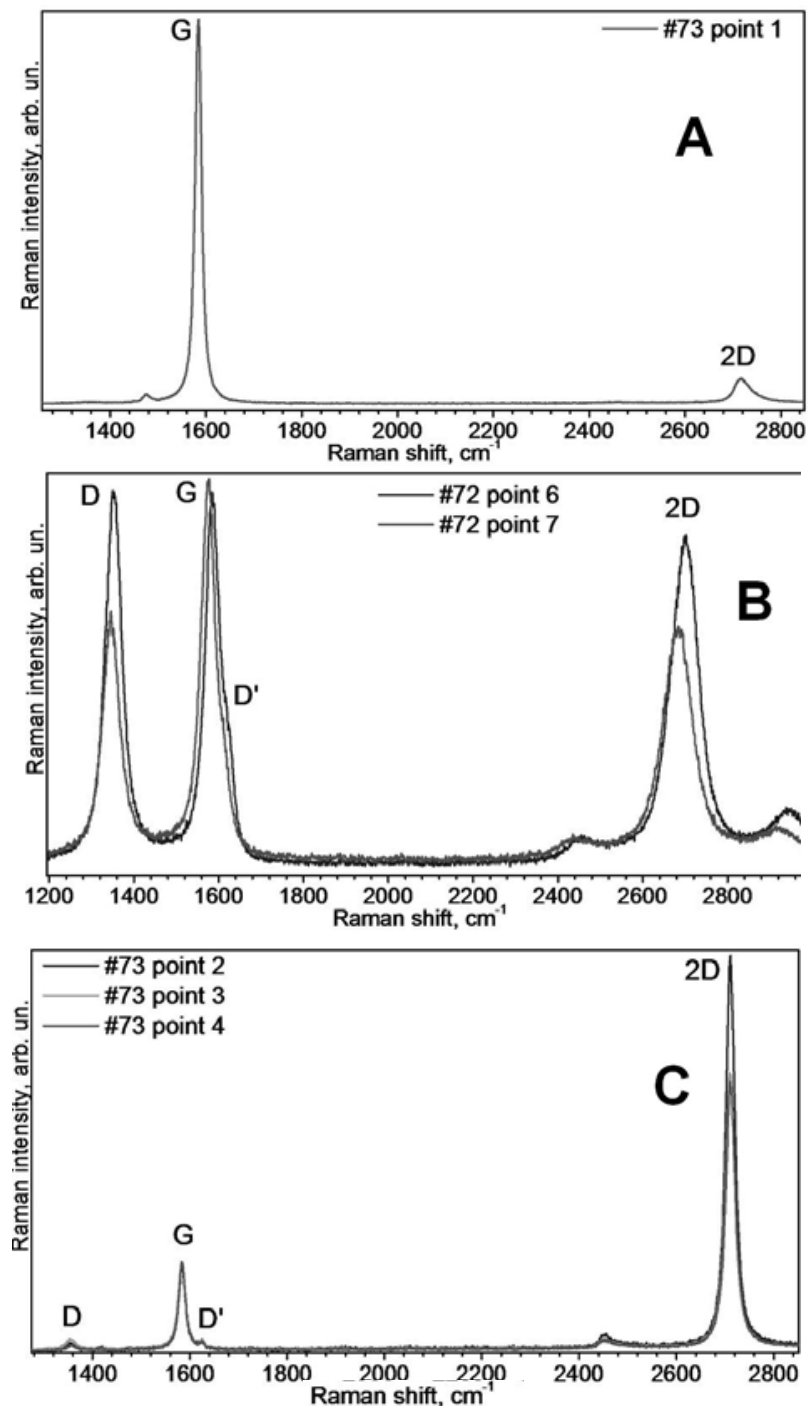

Fig. 3. Raman spectroscopy. A) Crystalline graphite; the spectrum has a high G-band amplitude and 2D doublet nature. B) Defective graphene layers, G- and D-bands can be observed in $\sim 1580 \mathrm{~cm}^{-1}$ and $\sim 1390 \mathrm{~cm}^{-1}$ regions; D-band, a structural defect presence indicator, 2D second order band is recorded at $\sim 2700 \mathrm{~cm}^{-1}$, which is conditioned by the presence of double electron and phonon resonance. C) One-layer graphene with high amplitude and 2D-band homogeneity, minor defective Dband amplitude is an evidence of a low number of defects. of which the capillary surfaces look varnished, surfaces of the high-temperature samples look ragged and flaky. The material is 'difficult' for photographing; colour shifts which are not observed directly appear on the pictures, and some details are not visible at all.

With 40× magnification and direct lighting with incandescent lamp, sharp contrasts are visible (Fig. 4C); these contrasts are also visible by the unaided eye in the form of almost black spaces between grey lines with metallic lustre. Using a powerful scattered light source forming soft shadows and a ring reflector, the contrasts disappear and all the sample areas are visible in shades of grey with cast-iron lustre (Fig. 1C).
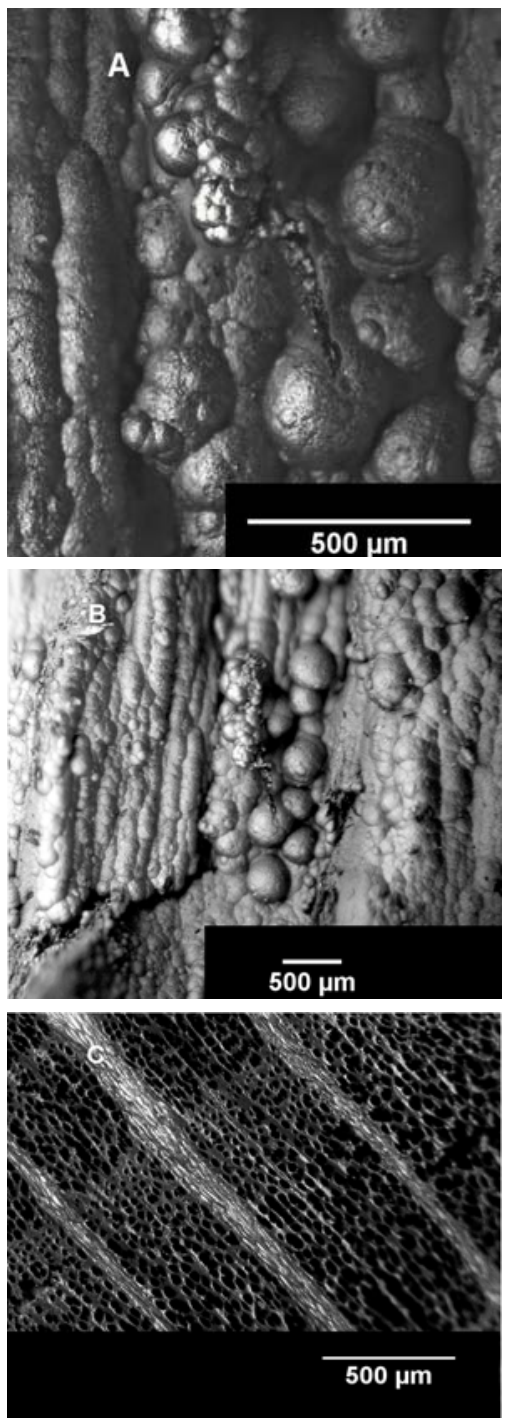

Fig. 4. Optical microscopy. A) External surfaces of the samples are covered with crystalline graphite bubbles; typical size of the bubbles is about $350 \mu \mathrm{m}$ and the smallest ones are about $60 \mu \mathrm{m}$. B) In the center of the photo one can see "bunch of grapes' grown on a hanging fiber as well as very extensive formations along the capillary channels. C) Transverse section after high-temperature processing observed under an incandescent lamp, transverse fibers glitter as metal, compaction of the structure in the middle of the sample is due to the wood growth yearly periods. 


\section{Results}

In the course of the studies, high quality graphenes were obtained from biomorphic materials and the following correlations were found.

Under pyrolysis temperature rise:

Increase in wettability with liquids and melts, carbon content and order degree, resistance to sharp temperature drops. Thus, when obtaining graphenes, rise of temperature was within the range $300 \ldots 400{ }^{\circ} \mathrm{C} / \mathrm{s}$.

The weight, size, electric resistance, content of impurities of hydrogen, nitrogen, oxygen and solid glassy bitumen are reduced.

The sequence of formation of graphites and graphenes is as follows: solid and gas-tight glassy carbon contained in the carbonized matrix is alloyed with potassium forming potassium acetylide. When the temperature rises, thermal plasticity of glassy carbon is activated, and when the potassium acetylide boiling temperature is reached, a sharp volume increase of the capsule takes place, its walls are stretched by pressure, and the reaction area increases; nitrogen, oxygen and hydrogen bonds break. This also explains the orbicular and spherical formations on the matrix surface as well as release of acetylene through the water barrier in the course of the experiment.

Due to high self-discharge current, it wasn't difficult to calculate capacitance that was about $4.5 \mathrm{~F} / \mathrm{cm}^{3}$ for the electrode material that is 17.7 times less than specific capacitance of industrial samples. Inner resistance was about $0.2 \mathrm{Ohm} / \mathrm{F}$, which corresponds to the industrial sample level.

Low specific capacitance is a weakness not for all applications but for energy storage systems only. When used as a filter for high frequencies and industrial interferences, low specific capacitance is not important.

It is known that among all types of nonpolar capacitors, the double electron layer ones have the worst frequency characteristics. It appears both as capacitance reduction is proportion to the frequency increase $[10,11]$, which results in growth of reactance and as significantly longer current rise time when the capacitor is charged or discharged. This property may be used as an inductivity substituting element as, like to that in the above-described capacitor, the inductivity reactance grows with the frequency increase, and the current rise rate is inversely proportional to the inductivity value. The double electron layer capacitor does not store energy in the same mode, in contrast to an inductive filter. This remarkable property prevents from occurrence of resonances.

The most important advantage of the two electron layer capacitor is the absence of voltage surges on cells of power units connected in-series with it, in contrast to a traditional industrial interference filter. This will allow building new well-protected power units and to protect already existing ones, reducing the breakage risk, raising soundness of any items connected to such power sources.
Another application is an audio power amplifier output stage that also works for the inductive load. Resonance quenching [11] in this case leads to significant sound reproduction quality improvement and acoustic fidelity increase.

The prospective possibility of construction of double electron layer capacitors using biomorphic carbon matrixes as electrodes has been proved. Capacitors built on graphene containing matrixes, due such key parameters as electric capacitance, are close to rechargeable batteries, and due to equivalent series resistance - to aluminum electric capacitors.

\section{Conclusions}

Graphene and graphite have the highest indices of tensile strength and high electrical conductivity. Graphite at high temperatures is one of the strongest materials. These properties allow making extremely durable carbon ceramics. The high electrical conductivity and developed surface are suitable for the manufacture from biomorphic graphene electrodes supercapacitors, batteries and accumulators with a high power level.

High-temperature pyrolysis of potassium-alloyed carbonized materials allows obtaining surfaces covered with nanostructures ranging from crystalline graphite to one-layer graphene with a low number of defects. The obtained graphenes have electric contacts with the matrix and are fixed on it; their linear sizes are huge and reach the entire length of the examined samples.

The biomorphic electrode-matrixes have a potential to exceed all the existing carbon electrodes by the key parameters.

If the further studies will prove the ability of acetylide-alloyed glassy carbon and solid anthracite-like bitumens to form monolithic extensive graphenes without dislocations, a real opportunity will occur to produce kilometers-long bands or macroscopic aprons by reeling of laser-generated, only one molecular layer thick products of desired length and width from an alloyed carbon cylinder.

\section{References}

1. V.S. Kiselov, V.A. Yukhymchyk, V.I. Poludin, M.P. Tryus, A.E. Belyaev, Mechanical properties of biomorphous ceramics // Semiconductor Physics, Quantum Electronics \& Optoelectronics, 15(4), p. 305 (2012).

2. P. Gao, M. Wu, B. Li, Y. Liu, Structure characterization and oxidation mechanism study of porous biomorphic carbon template derived from basswood // Mater. Res. Bull. 44, p. 644 (2009).

3. W. Dickson, Integrative Plant Anatomy. Academic Press, San Diego, CA, USA, 2000.

4. H. Sieber, C. Hoffmann, A. Kaindl, and P. Greil, Biomorphic cellular ceramics // Adv. Eng. Mater. 2(3), p. 105-109 (2000). 
5. M. Singh and J.A. Salem, Mechanical properties and microstructure of biomorphic silicon carbide ceramics fabricated from wood precursors // J. Eur. Ceram. Soc. 22, p. 2709 (2002).

6. A.I. Shelykh, B.I. Smirnov, T.S. Orlova, I.A. Smirnov, A.R. de Arellano-Lopez, J. Martinez-Fernandez, and F.M. Varela-Feria, Electrical and thermoelectric properties of the $\mathrm{SiC} / \mathrm{Si}$ biomorphic composite at high temperatures // Phys. Solid State, 48(2), p. 229 (2006).

7. J. Jiang, L. Zhang, X. Wang, N. Holm, K. Rajagopalan, F. Chen, Highly ordered macroporous woody biochar with ultra-high carbon content as supercapacitor electrodes // Electrochim. Acta, 113, p. 481 (2013).
8. V.D. Chekanova, A.S. Phialkov // Phys. Chem. 40(5), p. 777 (1971)

9. G.S. Rellick, D.J. Chang, and R.J. Zaldivar, Mechanisms of orientation and graphitization of hard-carbon matrices in carbon/carbon composites // Mechanics and Materials Technology Center, The Aerospace Corporation, El Segundo, California 90245-4691 (1992).

10. Electric Double Layer Capacitor: BOOSTCAP Module Datasheet, Maxwell Technologies, www.maxwell.com.

11. R.A. Dougal, L.Gao, S. Liu, Ultracapacitor model with automatic order selection and capacity scaling for dynamic system simulation // J. Power Sources, 126, p. 250 (2004). 